\title{
Caractères biogéochimiques de la matière organique dans la colonne d'eau et les sédiments d'un écosystème saumâtre: l'étang de Thau - Variations saisonnières Biogeochemical characters of organic matter in the water column and sediments of a brackish ecosystem: the Tau lagoon - Seasonal variations
}

\author{
L. Serve, F. Gadel, J. L. Lliberia et J. L. Blaz
}

Volume 12, numéro 4, 1999

URI : https://id.erudit.org/iderudit/705369ar

DOI : https://doi.org/10.7202/705369ar

\section{Aller au sommaire du numéro}

\section{Éditeur(s)}

Université du Québec - INRS-Eau, Terre et Environnement (INRS-ETE)

\section{ISSN}

0992-7158 (imprimé)

1718-8598 (numérique)

\section{Découvrir la revue}

Citer cet article

Serve, L., Gadel, F., Lliberia, J. L. \& Blaz, J. L. (1999). Caractères biogéochimiques de la matière organique dans la colonne d'eau et les sédiments d'un écosystème saumâtre: l'étang de Thau - Variations saisonnières. Revue des sciences de l'eau/ Journal of Water Science, 12(4), 619-642. https://doi.org/10.7202/705369ar

\section{Résumé de l'article}

Le long de la côte méditerranéenne française du Golfe du Lion, l'étang de Thau présente des caractères assez particuliers. Il est parfois soumis à des conditions anoxiques appelées "malaigues" qui résultent de l'accumulation de matières organiques durant la période chaude liée au développement des macrophytes. Ces dépôts organiques associés aux biomasses résultant des activités conchylicoles et aux apports extérieurs contribuent en cours d'année aux échanges biogéochimiques entre la colonne d'eau et les dépôts.

Dans ce même milieu, l'analyse de la distribution et de la nature de la matière organique par des méthodes fines comme la chromatographie liquide haute performance ou la pyrolyse a permis de préciser son origine et son évolution dans la colonne d'eau et les dépôts. Durant les quatre saisons, les particularités de la matière organique ont donc été analysées en terme d'accumulation, de dégradation et de conservation. L'été constitue une période de production et de dégradation. L'automne est principalement caractérisé par des processus dégradatifs et des apports terrigènes (composés phénoliques). L'hiver correspond à une période de relative stabilité de la matière organique consécutive aux conditions froides. Le printemps enfin représente une période de reprise de l'activité biologique produisant une matière organique fraîche riche en sucres.

Sous les tables conchylicoles on observe un accroissement de la matière organique dans la colonne d'eau et les dépôts. Mais les processus actifs de dégradation réduisent considérablement la quantité de matière organique déposée. Les résultats de ces mécanismes varient selon les stations sous table et hors table.

Dans les dépôts les résultats de la dégradation dans la colonne d'eau amènent à une décroissance des composés biodégradables et à un accroissemenet des composés résistants comme les phénols et les hydrocarbures aromatiques. Ces processus de minéralisation s'accroissent vers la profondeur dans les dépôts au profit du pôle aromatique.

Les relations entre les nutriments et la matière organique qui constitue à la fois leur source et leur puits se marquent bien sous les tables conchylicoles où les sels nutritifs s'accumulent en surface. 


\section{Caractères biogéochimiques de la matière organique dans la colonne d'eau et les sédiments d'un écosystème saumâtre : l'étang de Thau - Variations saisonnières}

- Biogeochemical characters of organic matter in the water column and sediments of a brackish ecosystem: the Thau lagoon - Seasonal variations

\section{SERVE ${ }^{*}{ }^{*}$, F. GADEL ${ }^{\dagger}, 2$, J.-L. LLIBERIA ${ }^{3}$ et J.-L. BLAZI ${ }^{1}$}

Reçu le 10 août 1998, accepté le 18 juillet 1999**

\section{SUMMARY}

The Thau lagoon along the French Mediterranean coast of the Gulf of Lions has unusual characteristics. It is sometimes subjected to anoxic conditions, known as "malaigues", which result from the accumulation of organic matter during the warmer period. Throughout the year this organic deposition, associated with oyster farming and terrigenous inputs, contributes to biogeochemical exchanges between the water column and the underlying deposits. In this same environment, high-resolution analytical techniques (HPLC; PY-GCMS) were used to analyze the distribution and nature of the organic matter and to determine its origin and behaviour in the water column and sediments.

Total suspended matter (TSM) was determined by filtration of water samples pumped up from different levels of the water column and filtered onto glass fiber filters (GF/F grade) previously heated at $450^{\circ} \mathrm{C}$ for 4 hours. Particulate organic carbon (POC) was determined on the same samples with a Leco CS 125 analyzer after removal of inorganic carbonates by treatment with a $\mathrm{H}_{2} \mathrm{SO}_{4}(2 \mathrm{~N})$ solution. Dissolved organic carbon (DOC) was determined on the filtrates using a Shimadzu TOC 5000 analyzer. The determination of polysaccharides in the TSM was achieved by a colorimetric method involving a $\mathrm{H}_{2} \mathrm{SO}_{4}$ (3N) solution and anthrone reagent (GALLALI, 1972).

Phenolic compounds were determined by high performance liquid chromatography (HPLC) after cupric oxide alkaline oxidation of TSM samples. The

1. Laboratoire de biologie physicochimique, Université de Perpignan, France.

2. CEFREM, Université de Perpignan, France.

3. Institut Quimic de Sarria, 08017 Barcelone, Espagne.

$\dagger$ In the memory of our friend and colleague François who passed away suddenly on April 25 of 1999 . His immense energies and his dedication to the subject of organic matter in lagunal environments will remain a model for us.

* Correspondance. E-mail : serve@univ-perp.fr

** Les commentaires seront reçus jusqu'au 15 novembre 2000. 
oxidized samples were acidified $(\mathrm{HCl}, 2 \mathrm{~N})$ and subjected to liquid-liquid extraction with ethyl acetate (HARTLEY \& BUCHAN 1973; HEDGES \& ERTEL 1982). The limit of detection is $10^{-4} \mathrm{~g}$ and the precision of the method is about $2 \%$ for each compound. Separation and quantification of phenolic monomers was carried out by HPLC (HARTLEY \& BUCHAN, 1973; SERVE et al., 1983). Of a total of 28 identified products, eleven represent the monomers constituting lignin and are taken into account according to HEDGES \& PARKER (1976), HEDGES \& MANN (1979) and HEDGES \& ERTEL (1982). The products of oxidative hydrolysis of lignin belong to the following three series: 4 hydroxybenzyl "H" (p-hydroxybenzoic acid, p-hydroxybenzaldehyde, phydroxyacetophenone), 3-methoxy-4-hydroxybenzylic "V" (Vanillyl) and 3,5-methoxy-4-hydroxybenzylic "S" (Syringyl). Each of these three series presents an alkyl side chain with 1,2 or 3 carbon atoms. The compounds in C6. $\mathrm{C} 1$ can be acids or aldehydes, those in $\mathrm{C6}-\mathrm{C} 2$ are ketones and those in $\mathrm{C6}-\mathrm{C3}$ are acids. The latter, having a phenylpropenic structure, belong to the Cinnamyl "C" series (ferulic acid, p-coumaric acid). Separation of phenols was carried out on a Merck analytical column ( $250 \mathrm{~mm}$ long $\times 4 \mathrm{~mm}$ in diameter) with a Lichrosorb reversed phase C18 stationary phase of $5 \mu \mathrm{m}$ granulometry, equipped with a precolumn ( $40 \mathrm{~mm}$ long) containing the same phase. Elution was achieved with ternary eluents (water, acetonitrile, acetic acid), in a high pressure binary gradient (CHARRIĚRE, 1991). The eluted products were determined qualitatively, by comparison of their retention times with those of commercial products (detection in $U V$ at $275 \mathrm{~nm}$ ), after a co-injection if necessary, and quantitatively by an internal standard method (phloroglucinol: 1,3,5-benzenetriol and p-anisic acid: p-methoxybenzoic acid).

Analysis of the major classes of organic compounds was carried out by coupled pyrolysis - gas chromatography - mass spectrometry. A CDS 1000 pyrolysis probe was directly fitted with a Perkin-Elmer 8700 gas chromatograph (GC) equipped with a TR-WAX capillary column (length: $30 \mathrm{~m}$, diameter: $0.32 \mathrm{~mm}$, phase thickness: $0.50 \mu \mathrm{m}$ ). Pyrolysis temperature was $700^{\circ} \mathrm{C}$ for $10 \mathrm{~s}$ and the column temperature was programmed from $60^{\circ} \mathrm{C}$ to $240^{\circ} \mathrm{C}$ at a rate of $6^{\circ} \mathrm{C} / \mathrm{min}$ according to PUIGBO et al. (1989). Pyrolysis fragments were identified by coupling the GC to a HP 5989 mass spectrometer. Twenty three major peaks were selected on the pyrochromatograms and each selected compound was expressed as a percentage of the sum of the surface of these 23 peaks Pyrolysis products were grouped into five main families, each of them including similar molecules or closely related chemical structures: aromatic hydrocarbons, nitrogenous compounds, sugars, phenols and amino sugars.

The survey of all these parameters showed some characteristic differences over the four seasons. Summer appears as a period where the biological production reaches maximum levels in the water column. At that time, organic matter is stratified with high levels of accumulation in the deeper layers. DOC is also abundant throughout the water column and organic compounds belonging to the class of sugars decrease according to depth. Autumn corresponds to Mediterranean storms and typical rainfalls. Terrestrial inputs increase in this season and degradative processes affect the organic matter that was produced in large quantities in the summer by the autotrophic organisms of the lagoon. DOC is recycled and reflects the degradation of autochthonous organic material. Winter, with reduced TSM levels related to low terrestrial inputs, is characterized by a homogenization of the water column and a weak biological activity. Lignin-derived phenols are abundant and correspond to a period of low biological activity. In contrast, in the spring the biological activity recovers, as indicated by the high sugar content of the DOC and by a homogenization of the water column.

Under the oyster beds, an increase of organic matter is observed in the water column as well as in the sediments. However, the active degradation processes in summer and autumn reduce considerably the amount of the settling orga- 
nic matter. The results of these processes are variable according to whether the stations are under or outside of the oyster beds. Degradation in the water column leads to a decrease of biodegradable compounds in the sediments and an increase in resistant compounds like phenols and aromatic hydrocarbons. These mineralization processes increase with depth in deposits, as reflected by higher proportions of aromatic compounds. The relationship between nutrients and organic matter, the latter constituting both their source and their sink, appears in sediments under oyster beds, where the inorganic nutrients accumulate at the surface.

Key-words: biogeochemistry, organic matter, lagoons, Mediterranean, gulf of Lions, seasonal variations.

Le long de la côte méditerranéenne française du Golfe du Lion, l'étang de Thau présente des caractères assez particuliers. Il est parfois soumis à des conditions anoxiques appelées * malaigues » qui résultent de l'accumulation de matières organiques durant la période chaude liée au développement des macrophytes. Ces dépôts organiques associés aux biomasses résultant des activités conchylicoles et aux apports extérieurs contribuent en cours d'année aux échanges biogéochimiques entre la colonne d'eau et les dépôts.

Dans ce même milieu, P'analyse de la distribution et de la nature de la matière organique par des méthodes fines comme la chromatographie liquide haute performance ou la pyrolyse a permis de préciser son origine et son évolution dans la colonne d'eau et les dépôts. Durant les quatre saisons, les particularités de la matière organique ont donc été analysées en terme d'accumulation, de dégradation et de conservation. L'été constitue une période de production et de dégradation. L'automne est principalement caractérisé par des processus dégradatifs et des apports terrigènes (composés phénoliques). L'hiver correspond à une période de relative stabilité de la matière organique consécutive aux conditions froides. Le printemps enfin représente une période de reprise de l'activité biologique produisant une matière organique fraîche riche en sucres.

Sous les tables conchylicoles on observe un accroissement de la matière organique dans la colonne d'eau et les dépôts. Mais les processus actifs de dégradation réduisent considérablement la quantité de matière organique déposée. Les résultats de ces mécanismes varient selon les stations sous table et hors table.

Dans les dépôts les résultats de la dégradation dans la colonne d'eau amènent à une décroissance des composés biodégradables et à un accroissemenet des composés résistants comme les phénols et les hydrocarbures aromatiques. Ces processus de minéralisation s'accroissent vers la profondeur dans les dépôts au profit du pôle aromatique.

Les relations entre les nutriments et la matière organique qui constitue à la fois leur source et leur puits se marquent bien sous les tables conchylicoles où les sels nutritifs s'accumulent en surface.

Mots clés : biogéochimie, matière organique, lagunes, Méditerranée, golfe du Lion, variations saisonnières. 


\section{1 - INTRODUCTION}

Le long du Golfe du Lion les étangs littoraux forment un chapelet continu depuis l'embouchure du Rhône jusqu'à la côte espagnole et constituent des milieux intermédiaires entre le continent et le domaine marin et, à ce titre, des écosystèmes tampons dans les rapports entre ces deux domaines (SENEGAS et DOMANGE 1990). Les environnements lagunaires méditerranéens se caractérisent par des perturbations dystrophiques saisonnières appelées " malaigues" en rapport avec le développement de conditions anoxiques résultant de l'accumulation des matières organiques quand la température atteint des niveaux très élevés en été (développement des macrophytes) (FRISONI et CEJPA 1985).

Dans l'étang de Thau, en Languedoc-Roussillon, (surface $75 \mathrm{~km}^{2}$ et profondeur moyenne $5 \mathrm{~m}$, figure 1), ces perturbations trophiques semblent dépendre des conditions climatiques et éventuellement du développement extensif de l'ostréiculture (production supérieure à 15000 t/an, DESLOUS-PAOLI et al. 1991). La dégradation de la végétation initiale, particulièrement d'Ulva lactuca (Chloro-

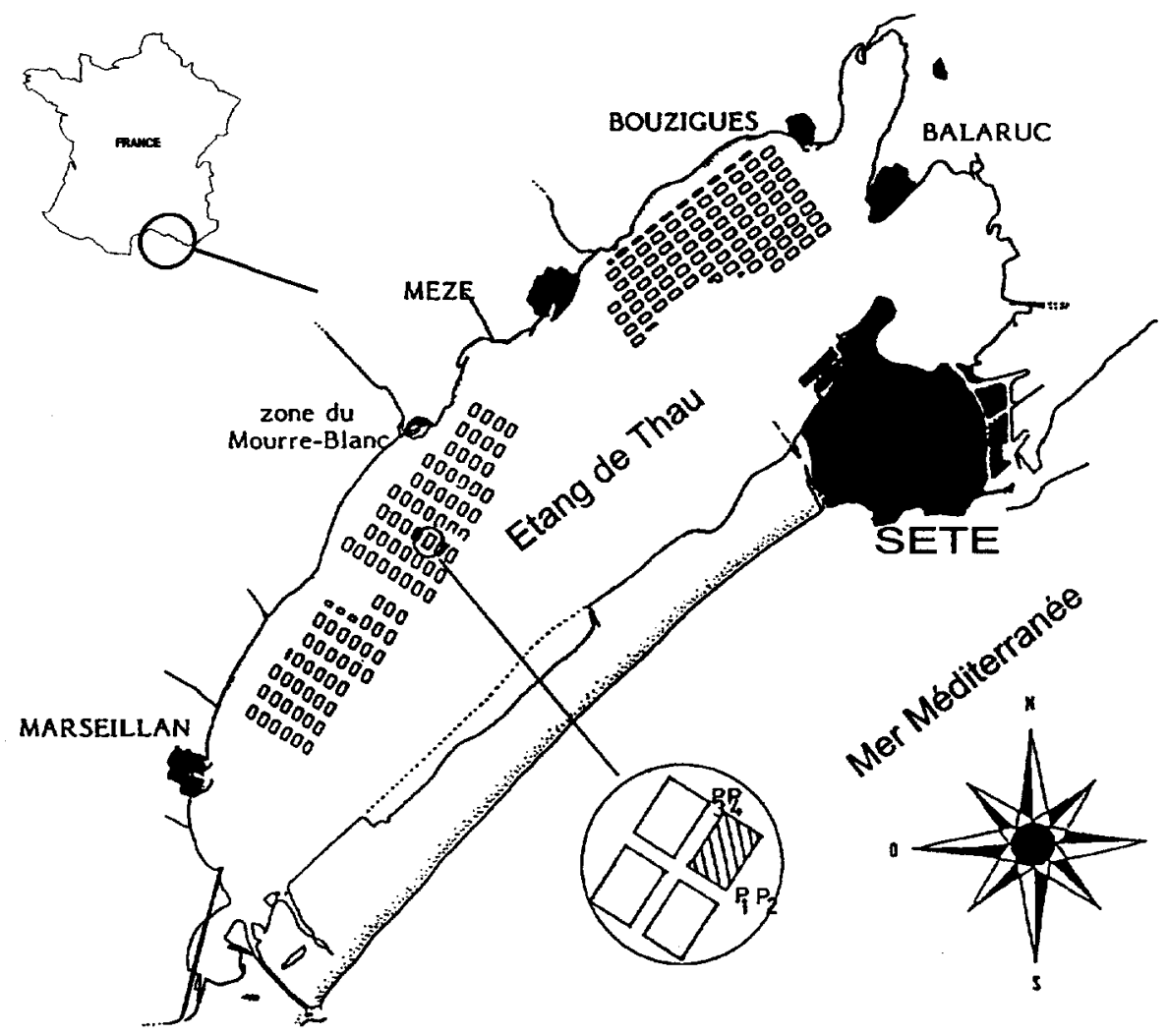

Figure 1 Localisation des stations d'étude dans l'étang de Thau (colonne d'eau : P1, P2, P3 et P4-dépôts : S1 et S2).

Location of the sampling stations in the Thau lagoon (water column: $P 1, P 2, P 3$ and P4 - deposits: S1 and S2). 
phyceae) constitue également un facteur important dans le démarrage des crises dystrophiques.

Le programme OXYTHAU, qui a suivi le programme ECOTHAU, s'est développé de 1991 à 1995. Ces actions ont permis une description des paramètres physicochimiques et biologiques et de l'hydrodynamisme de l'écosystème Thau (MILLET, 1989 ; JOUFRE et AMANIEU, 1991 ; SERVE, et al. 1993 ; GADEL, 1994 ; GADEL et SERVE, 1994 ; MESNAGE, 1994). Les objectifs majeurs de ces études étaient d'évaluer l'impact de l'ostréiculture sur ce milieu à travers la quantification des flux de matière aux interfaces eau-sédiment et eau-organismes filtreurs. Le bilan de l'azote et du phosphore a été précisé et la modélisation des cycles biogéochimiques de ces éléments réalisée (BIOTEAU, 1993 ; CHAPELLE, 1993).

Aussi, il paraît important de mieux préciser l'impact du climat et de l'ostréiculture sur la sédimentation organique dans la colonne d'eau, ceci sur différents sites sélectionnés dans la lagune. L'étude quantitative et qualitative de la matière organique comprise dans les flux de matières en suspension et dans les dépôts doit permettre de déterminer son impact véritable dans les processus d'eutrophisation avant d'établir un modèle fonctionnel et de connaître ses relations avec la distribution des nutriments.

\section{2 - MATÉRIEL ET MÉTHODES}

L'étude a concerné quatre stations qui se situent sous et à proximité des tables à huitres ( 3 sous table: P1, P2, P3 et 1 hors table : P4) (figure 1). Les eaux ont été prélevées par pompage au cours des quatre saisons, de l'été 91 au printemps 92 (juillet et octobre 1991 ; janvier et avril 1992), ceci à cinq profondeurs de la colonne d'eau: surface, 1, 2, 3 et 4 mètres de profondeur. Elles ont été filtrées sur filtres GFF (Whatman) préalablement brulés afin de séparer la matière en suspension de la matière en solution. Les matières en supension ont ensuite été lyophilisées.

Les sédiments ont été prélevés en plongée à l'aide de carottiers $(30 \mathrm{~cm}$ de long et $6 \mathrm{~cm}$ de diamètre) sur deux stations, sous table (S1) et hors table (S2) (figure 1). Les dépôts ont été découpés en tranches de $5 \mathrm{~cm}$ jusqu'à $25 \mathrm{~cm}$ de profondeur. Après congélation elles ont été lyophilisées

Le carbone organique particulaire (COP) a été analysé par combustion sèche dans les filtres et les sédiments après décarbonatation par $\mathrm{HCl} 2 \mathrm{~N}$ avec un analyseur LECO CS 125.

Le carbone organique dissous (COD) a été analysé dans les eaux après filtration sur filtres Whatman GFF avec un appareil TOC 5000 Shimadzu. Toutefois cette filtration, de par la taille des pores des filtres de l'ordre de 0,45 $\mu \mathrm{m}$, ne permet pas d'obtenir la fraction dissoute réelle. Les échantillons d'eau ont été stérilisés avec une solution de $\mathrm{HgCl}_{2}\left(\left.1 \mathrm{~g} \cdot\right|^{-1}\right)$ immédiatement après filtration.

L'analyse des polysaccharides a été effectuée dans la phase particulaire (suspensions et dépôts) par colorimétrie avec le réactif anthrone en prenant le glucose comme standard, selon GALLALI (1972). 
La méthode d'analyse des composés phénoliques adoptée est l'oxydation alcaline par l'oxyde cuivrique des suspensions et des dépôts lyophilisés. La séparation et le dosage des monomères phénoliques ont été réalisés par chromatographie liquide haute performance CLHP (HARTLEY et BUCHAN, 1979 ; SERVE et al., 1983). Onze monomères constitutifs de la lignine ont été analysés selon HEDGES et.ERTEL (1982). Les produits de l'hydrolyse oxydative de la lignine appartiennent aux trois séries : 4-Hydroxybenzylique $(H)$, Vanillique $M$ et Syringique (S) ainsi qu'à la série Cinnamique (C) (SERVE et al., 1997).

La séparation des phénols a été réalisée sur une colonne analytique Merk (250 $\mathrm{mm}$ de long par $4 \mathrm{~mm}$ de diamètre). La phase stationnaire est constituée de Lichrosorb RP-18 de granulométrie $5 \mu \mathrm{m}$ (avec une précolonne de $40 \mathrm{~mm}$ de longueur contenant la même phase). L'élution se fait avec un mélange ternaire : eau, acétonitrile et acide acétique suivant un gradient binaire haute pression défini par CHARRIERE et al., (1986). L'identification des produits élués a été effectuée par comparaison de leur temps de rétention avec ceux de produits commerciaux, la détection se faisant dans l'UV à $275 \mathrm{~nm}$ et leur quantification par étalonnage interne (phloroglucinol et acide p-anisique).

L'analyse des classes majeures de composés organiques par pyrolyse-chromatographie en phase gazeuse-spectrométrie de masse dans les suspensions (stations P2 et P4) et les dépôts a été réalisée avec une pyrosonde CDS 190 directement couplée à un chromatographe en phase gazeuse Perkin Elmer 8700 équipé d'une colonne capillaire TR-WAX de $30 \mathrm{~m}$ de long par $0,32 \mathrm{~mm}$ de diamètre intérieur et une épaisseur de film de 0,50 $\mu \mathrm{m}$ (Institut Quimic de Sarria-Barcelone). La pyrolyse s'effectue à $700^{\circ} \mathrm{C}$ durant $10 \mathrm{~s}$ avec une température de chauffe de $10^{\circ} \mathrm{C} \mathrm{ms}-1$. La température est progressivement montée de 50 à $240^{\circ} \mathrm{C}$ à une vitesse de $6^{\circ} \mathrm{C} \mathrm{min-1.} \mathrm{On} \mathrm{utilise} \mathrm{la} \mathrm{détection} \mathrm{par} \mathrm{ionisa-}$ tion de flamme avec l'hélium comme gaz vecteur. Les produits dérivés de la pyrolyse sont identifiés par couplage avec un spectromètre de masse quadrupole HP 5899 (ionisation d'impact d'électrons $70 \mathrm{eV}$ ) par comparaison avec des pyrogrammes similaires analysés antérieurement. 23 pics majeurs sont sélectionnés et leur somme rapportée à $100 \%$ (PUIGBO et al., 1989). Cinq familles de composés organiques sont ainsi séparées : 1- Hydrocarbures aromatiques (benzène, toluène et styrène), 2- Composés azotés (acétonitrile, valéronitrile, pyridine, pyrrole, 3 et 2 méthyl pyrrole, benzylonitile et indole), 3- Sucres (cyclopentenone, 3 furanaldéhyde, acide acétique, furfural, 5 méthyl 2-furfural, 2-3 dihydrobenzofurane), 4- Phénols (phénol, $p$ et $m$-crésols, éthylphenol et phényléthanol) et 5- Sucres aminés (acétamide). Les teneurs sont exprimées en pourcentages relatifs.

\section{3 - RÉSULTATS ET DISCUSSION}

\subsection{Analyse de la colonne d'eau}

\subsubsection{Les matières en suspension (MES)}

Les variations saisonnières des MES résultent de l'importance relative des fractions organique et minérale (figure 2). Le mois de juillet est caractérisé par 


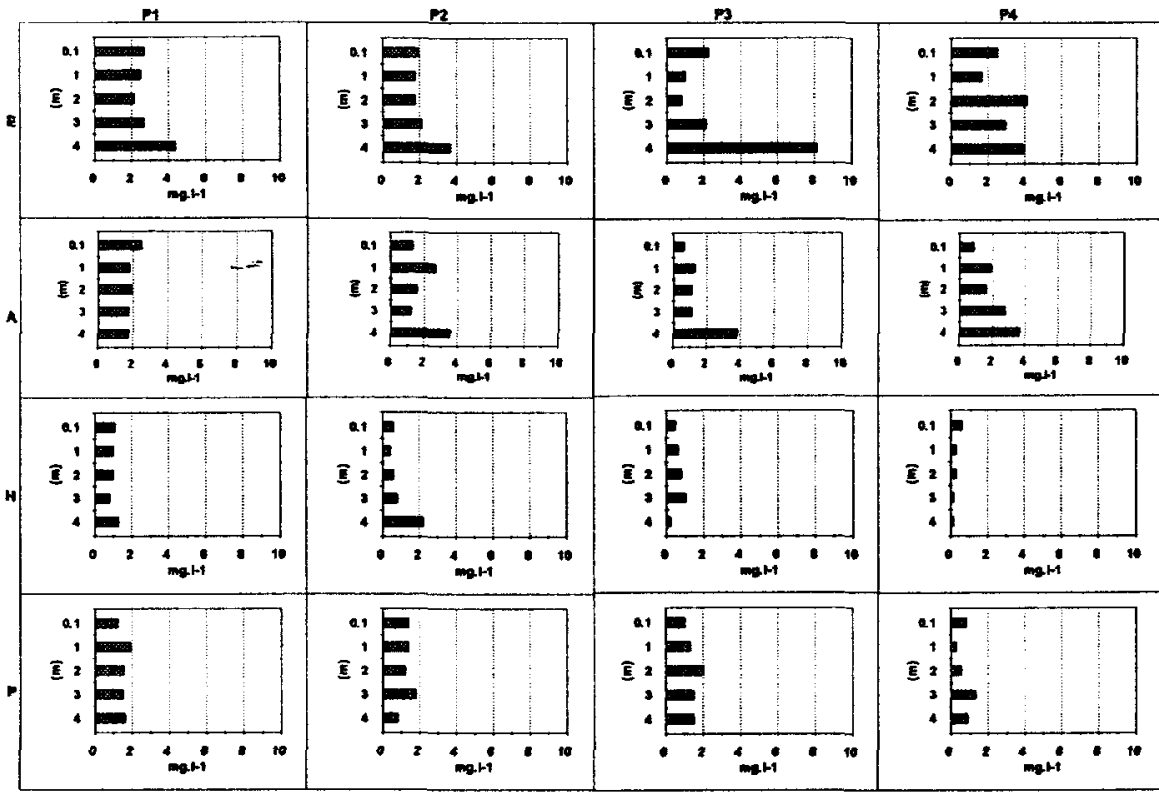

Figure 2 Évolution de la charge en suspension $\left(\mathrm{mg} \cdot \mathrm{r}^{-1}\right.$ ) dans la colonne d'eau au cours des saisons (E, $A, H$ et $P)$ sur les quatre stations sélectionnées.

Seasonal changes in concentrations of suspended matter $\left(\mathrm{mg} \cdot \mathrm{r}^{-1}\right)$ in the water column at the four selected stations $(E=$ summer, $A=$ autumn, $H=$ winter, $P=$ spring).

l'abondance de la matière organique dans les MES (moyenne 2,5 mg/l) en rapport avec la forte activité biologique (algues, macrophytes...). Le mois d'octobre montre des valeurs légérement moindres (moyenne $1,9 \mathrm{mg} \cdot \mathrm{l}^{-1}$ ). En janvier, les valeurs, relativement basses (moyenne $0,9 \mathrm{mg} \cdot \mathrm{H}^{-1}$ ), sont à rapporter à la baisse hivernale de l'activité biologique. Au contraire, en avril, la reprise de cette activité entraîne un léger accroissement des MES (moyenne $1,4 \mathrm{mg} \cdot{ }^{-1}$ ) et de la charge organique en suspension.

Des variations sont observées en été et en automne dans la colonne d'eau. L'accroissement des MES avec la profondeur en été est probablement dû aux processus de décantation progressive plutôt qu'à une remise en suspension, compte tenu du faible développement des courants qui sont par ailleurs freinés par les tables.

\subsubsection{Le carbone organique particulaire (COP) et le carbone organique dissous (COD) - Rapport COD/COP}

Les variations du COP $\left(\mathrm{mg} \cdot \mathrm{I}^{-1}\right)$ peuvent être directement comparées à celles du MES et sont tout à fait parallèles (figure 3). Elles résultent essentiellement des conditions climatiques méditerranéennes, de l'activité biologique, du développement de la végétation, des pluies qui interviennent en automne et au printemps. Les valeurs du COP sont élevées en juillet (activité biologique) et en 
P1

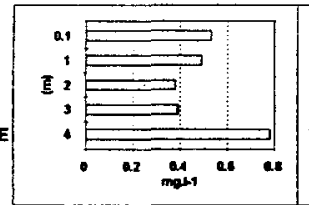

P2

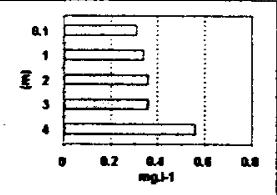

P3

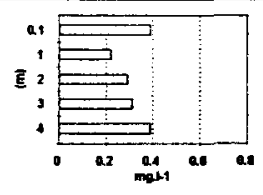

P4

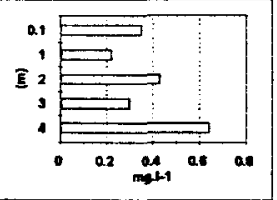

$\ldots$
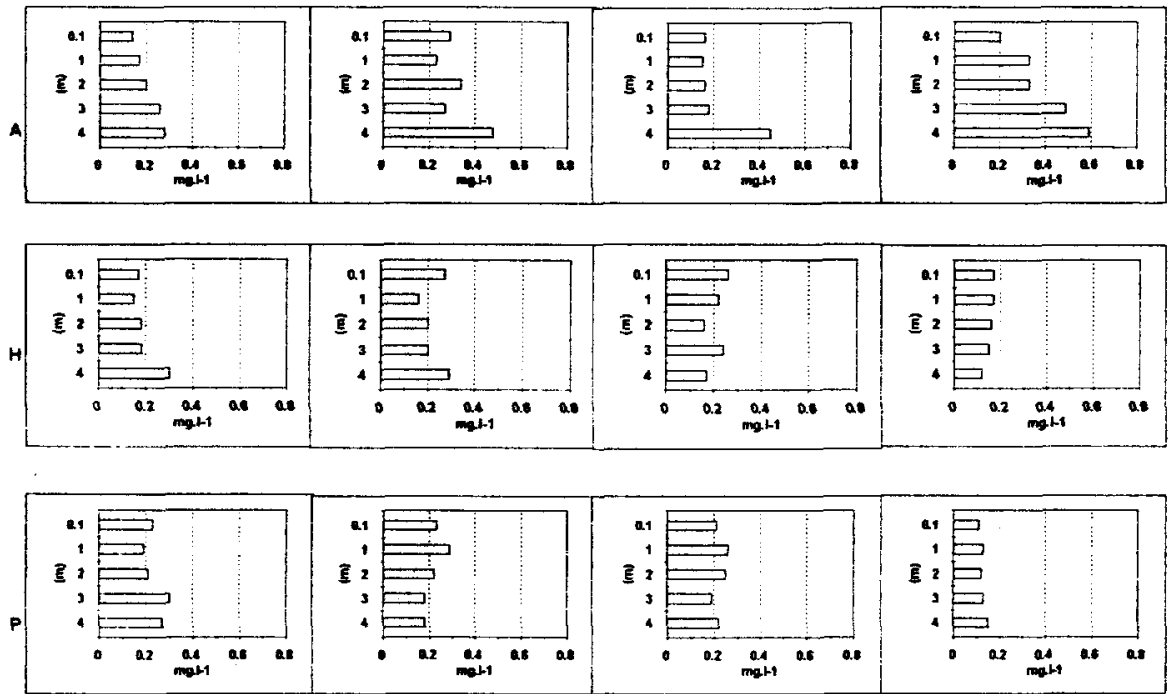

Figure 3 Évolution des teneurs en $\operatorname{COP}\left(\mathrm{mg} \cdot \mathrm{l}^{-1}\right)$ dans la colonne d'eau au cours des saisons ( $E, A, H$ et $P$ ) sur les quatre stations sélectionnées.

Seasonal changes in concentrations of particulate organic carbon ( $m g$ POC $r^{-1}$ ) in the water column at the four selected stations ( $E=$ summer, $A=$ autumn, $H=$ winter, $P=$ spring).

octobre. Elles présentent un minimum en janvier, plus particulièrement à la station P4 située hors des tables. Elles s'accroissent légèrement en avril au moment où intervient la reprise de l'activité biologique.

Les valeurs du COP $\left(\mathrm{mg} \cdot \mathrm{l}^{-1}\right)$ comme celles du MES s'accroissent en profondeur en juillet et en octobre, probablement dues aux processus de décantation du fait de l'absence de courants.

Si les teneurs en carbone organique particulaire (COP \%) dans les suspensions sont plus élevées en automne, au printemps et surtout en été (de 7 à $38 \%)$, elles décroissent en hiver, traduisant la forte réduction de l'activité biologique (de 1 à 7,7 ) (tableau 1).

Comme pour le COP, les teneurs en COD les plus fortes avec des valeurs extrêmes jusqu'à plus de $30 \mathrm{mg} \cdot \mathrm{I}^{-1}$ s'observent en juillet, période de forte dégradation de la matière organique particulaire et de processus actifs d'excrétion des organismes vivants (tableau 2). Elles sont beaucoup plus faibles durant les autres périodes (de 2,9 à $3,8 \mathrm{mg} \cdot \mathrm{l}^{-1}$ ). 
Tableau 1 Teneurs en carbone organique particulaire (\%) dans les suspensions sur les quatre stations et à cinq profondeurs de la colonne d'eau.

Table 1 Particulate organic contents (\%) in suspended material at the four stations and at five depths.

\begin{tabular}{|c|c|c|c|c|c|c|c|c|}
\hline & \multicolumn{4}{|c|}{ Juillet 91} & \multicolumn{4}{|c|}{ Janvier 92} \\
\hline & P1 - & $\mathbf{P 2}$ & P3 & P4 & P1 & P2 & P3 & P4 \\
\hline $0,1 \mathrm{~m}$ & 19,6 & 12,4 & 2,5 & 19,3 & 17,7 & 26,7 & 4,6 & 12,7 \\
\hline $1 \mathrm{~m}$ & 19,6 & 9,4 & 2,3 & 11,6 & 22 & 11,5 & 4 & 17,6 \\
\hline $2 m$ & 18,1 & 10,5 & 3,3 & 13,8 & 36,2 & 14,5 & 3,9 & 15,1 \\
\hline $3 m$ & 15 & 15,3 & 2,3 & 19,3 & 14,8 & 16,4 & 2,8 & 10,4 \\
\hline \multirow[t]{3}{*}{$4 m$} & 18,1 & 16 & 2,1 & 20,9 & 48,1 & 11,8 & 5,6 & 12,7 \\
\hline & \multicolumn{4}{|c|}{ Octobre 91} & \multicolumn{4}{|c|}{ Avril 92} \\
\hline & P1 & $\mathbf{P} 2$ & P3 & P4 & P1 & P2 & P3 & P4 \\
\hline $0,1 \mathrm{~m}$ & 16,3 & 21,5 & 7,7 & 20,9 & 6,7 & 23,5 & 1,5 & 11,3 \\
\hline $1 \mathrm{~m}$ & 20 & 8,4 & 3 & 20,2 & 13,7 & 17,4 & 4,3 & 17,5 \\
\hline $2 m$ & 17,6 & 21,2 & 4 & 19,7 & 10,5 & 20,6 & 3,9 & 8,7 \\
\hline $3 \mathrm{~m}$ & 17,1 & 22,5 & 5,8 & 8 & 17,2 & 17,8 & 4,6 & 20 \\
\hline $4 m$ & 15,6 & 13,5 & 1,1 & 27,5 & 16,4 & 16,2 & 1,8 & 37,7 \\
\hline
\end{tabular}

Tableau 2 Teneurs en carbone organique dissous $\left(\mathrm{mg} \cdot \mathrm{f}^{-1}\right)$ sur les quatre stations et à cinq profondeurs de la colonne d'eau.

Table 2 Dissolved organic carbon contents $\left(m g . r^{-1}\right)$ at the four stations and at five depths.

\begin{tabular}{|c|c|c|c|c|c|c|c|c|}
\hline & \multicolumn{4}{|c|}{ Juillet 91} & \multicolumn{4}{|c|}{ Janvier 92} \\
\hline & P1 & P2 & P3 & P4 & P1 & $\mathbf{P 2}$ & P3 & P4 \\
\hline $0,1 \mathrm{~m}$ & 7,6 & 140 & 56,5 & 6,4 & 2,7 & 3,1 & 3,4 & 3,2 \\
\hline $1 \mathrm{~m}$ & 6,6 & 4,9 & 4,4 & 4,6 & 2,9 & 77 & 3,7 & 8,2 \\
\hline $2 m$ & 15,9 & 5,3 & 3,6 & 3,7 & 2,9 & 3 & 3,6 & 8,3 \\
\hline $3 \mathrm{~m}$ & 4,6 & 4,3 & 12,2 & 4,4 & 3,2 & 2,7 & 3,9 & 2,8 \\
\hline \multirow[t]{3}{*}{$4 \mathrm{~m}$} & 16 & 40,6 & 4,8 & 150 & 5,3 & 2,9 & 3 & 2,5 \\
\hline & \multicolumn{2}{|c|}{ Octobre 91} & & & \multicolumn{4}{|c|}{ Avril 92} \\
\hline & P1 & P2 & P3 & P4 & P1 & $\mathbf{P 2}$ & P3 & P4 \\
\hline $0,1 \mathrm{~m}$ & 2,9 & 2,9 & 2,9 & 3,2 & 2,8 & 2,8 & 3,5 & 2,7 \\
\hline $1 \mathrm{~m}$ & 3,1 & 3,3 & 2,9 & 3,2 & 2,8 & 2,7 & 2,6 & 2,8 \\
\hline $2 m$ & 3,1 & 3,4 & 3 & 3,2 & 2,8 & 2,7 & 2,6 & 2,6 \\
\hline $3 m$ & 3,9 & 3,2 & 3,1 & 3,4 & 3,5 & 3 & 2,9 & 2,8 \\
\hline $4 m$ & 3,1 & 3,4 & 3 & 3,5 & 3,4 & 2,7 & 3,2 & 2,7 \\
\hline
\end{tabular}

Les valeurs les plus fortes du rapport COD/COP et quelquefois extrêmes jusqu'à 300 s'observent en juillet (moyenne 59), les plus faibles en octobre 
Tableau 3 Valeurs du rapport COD/COP sur les quatre stations et à cinq profondeurs de la colonne d'eau.

Table $3 \quad C O D / C O P$ values at the four stations and at five depths.

\begin{tabular}{|c|c|c|c|c|c|c|c|c|}
\hline & \multicolumn{4}{|c|}{ Juillet 91} & \multicolumn{4}{|c|}{ Janvier 92} \\
\hline & P1 & P2 & P3 & P4 & P1 & P2 & P3 & P4 \\
\hline $0,1 \mathrm{~m}$ & 14,3 & 542,6 & 144,9 & 18,3 & 15,9 & 11,5 & 13,1 & 18,8 \\
\hline $1 \mathrm{~m}$ & 13,5 & 14,4 & 19,6 & 20,9 & 19,3 & 482,5 & 16,8 & 18,2 \\
\hline $2 m$ & 41,8 & 17,7 & 12,4 & 8,6 & 16,1 & 15,0 & 22,5 & 51,9 \\
\hline $3 \mathrm{~m}$ & 11,8 & 11,9 & 12,3 & 8,8 & 17,8 & 13,5 & 17,7 & 18,7 \\
\hline \multirow[t]{3}{*}{$4 m$} & 20,5 & 72,5 & 39,4 & 275,8 & 17,7 & 10,0 & 16,3 & 20,8 \\
\hline & \multicolumn{2}{|c|}{ Octobre 91} & & & \multicolumn{4}{|c|}{ Avril 92} \\
\hline & $P 1$ & P2 & P3 & $\mathbf{P 4}$ & P1 & $\mathbf{P 2}$ & P3 & P4 \\
\hline $0,1 \mathrm{~m}$ & 20,7 & 10,0 & 18,1 & 16,0 & 12,2 & 12,2 & 16,7 & 24,6 \\
\hline $1 \mathrm{~m}$ & 18,2 & 14,4 & 19,3 & 9,7 & 14,7 & 9,3 & 10,0 & 21,5 \\
\hline $2 \mathrm{~m}$ & 15,5 & 10,0 & 18,8 & 10,3 & 13,3 & 12,3 & 10,4 & 21,7 \\
\hline $3 \mathrm{~m}$ & 15,0 & 11,9 & 6,7 & 6,9 & 11,7 & 16,7 & 14,6 & 21,5 \\
\hline $4 m$ & 11,1 & 7,1 & 17,2 & 5,9 & 12,6 & 15,0 & 15,3 & 18,0 \\
\hline
\end{tabular}

Tableau 4 Teneurs en polysaccharides $\left(\mathrm{mg} \cdot{ }^{-1}\right)$ dans les suspensions sur les quatre stations et à cinq profondeurs de la colonne d'eau.

Table 4 Polysaccharide contents $\left(\mathrm{mg} \cdot \mathrm{r}^{-1}\right)$ in suspended material at the four stations and at five depths.

\begin{tabular}{|c|c|c|c|c|c|c|c|c|}
\hline & \multicolumn{4}{|c|}{ Juillet 91} & \multicolumn{4}{|c|}{ Octobre 91} \\
\hline & P1 & $\mathbf{P 2}$ & P3 & P4 & P1 & $\mathbf{P 2}$ & P3 & P4 \\
\hline $0,1 \mathrm{~m}$ & 120 & 80 & 120 & 100 & 50 & 140 & 30 & 140 \\
\hline $1 \mathrm{~m}$ & 190 & 60 & 70 & 60 & 40 & 90 & 40 & 130 \\
\hline $2 m$ & 110 & 90 & 60 & 100 & 50 & 70 & 300 & 110 \\
\hline $3 \mathrm{~m}$ & 100 & 80 & 70 & 100 & 60 & 70 & 90 & 110 \\
\hline \multirow[t]{3}{*}{$4 m$} & 150 & 110 & 80 & 130 & 70 & 80 & 120 & 290 \\
\hline & \multicolumn{4}{|c|}{ Janvier 92} & \multicolumn{4}{|c|}{ Avril 92} \\
\hline & P1 & $\mathbf{P 2}$ & $\mathbf{P 3}$ & P4 & P1 & P2 & $\mathbf{P 3}$ & P4 \\
\hline $0,1 \mathrm{~m}$ & 60 & 60 & 80 & 40 & 120 & 125 & 85 & 50 \\
\hline $1 \mathrm{~m}$ & 40 & 40 & 30 & 40 & 70 & 75 & 70 & 45 \\
\hline $2 m$ & 50 & 50 & 30 & 30 & 80 & 95 & 60 & 40 \\
\hline $3 \mathrm{~m}$ & 40 & 40 & 30 & 40 & 180 & 110 & 125 & 42 \\
\hline $4 \mathrm{~m}$ & 50 & 90 & 25 & 40 & 135 & 65 & 55 & 75 \\
\hline
\end{tabular}

(moyenne 13) (tableau 3). Elles s'accroissent en janvier (moyenne 39) et diminuent en avril (moyenne 15). En période estivale, les effets de dégradation très actifs peuvent favoriser le passage de la forme particulaire à la forme dissoute fortement dégradée au cours de la période automnale. 


\subsubsection{Les polysaccharides}

Les teneurs en polysaccharides constituent un paramètre important directement en rapport avec la productivité locale des organismes phytoplanctoniques et, en conséquence, sont plutôt indicatrices d'une origine autochtone (WALSH, 1966). Toutefois, les apports terrigènes riches en cellulose peuvent aussi accroître les valeurs en automne (tableau 4). Un accroissement des valeurs $\left(\mathrm{mg} \cdot \mathrm{l}^{-1}\right)$ est observé vers la profondeur en été et en automne dans la colonne d'eau (jusqu'à $0,29 \mathrm{mg} \cdot \mathrm{l}^{-1}$ ), corrélé avec l'augmentation des valeurs du COP (décantation progressive de la matière organique).

Élevées en été et un peu moindres en automne, les valeurs montrent une importante baisse en hiver dans les suspensions $\left(0,05 \mathrm{mg} \mathrm{l}^{-1}\right)$, suivie par un léger accroissement printanier $\left(0,1 \mathrm{mg} \mathrm{l}^{-1}\right)$ (tableau 4). Des teneurs plus fortes caractérisent la station P4 hors table en automne, mais sont plus basses en janvier.

Les valeurs du carbone des polysaccharides rapportées au carbone organique particulaire (\%) sont moyennes en été, en automne et en hiver (figure 4). Un accroissement est observé au printemps, probablement dû à la reprise de l'activité biologique (fraîcheur du matériel). La décroissance assez évidente avec la profondeur en été et en automne sur la plupart des stations traduit une forte dégradation de ces composés biodégradables dans la colonne d'eau.

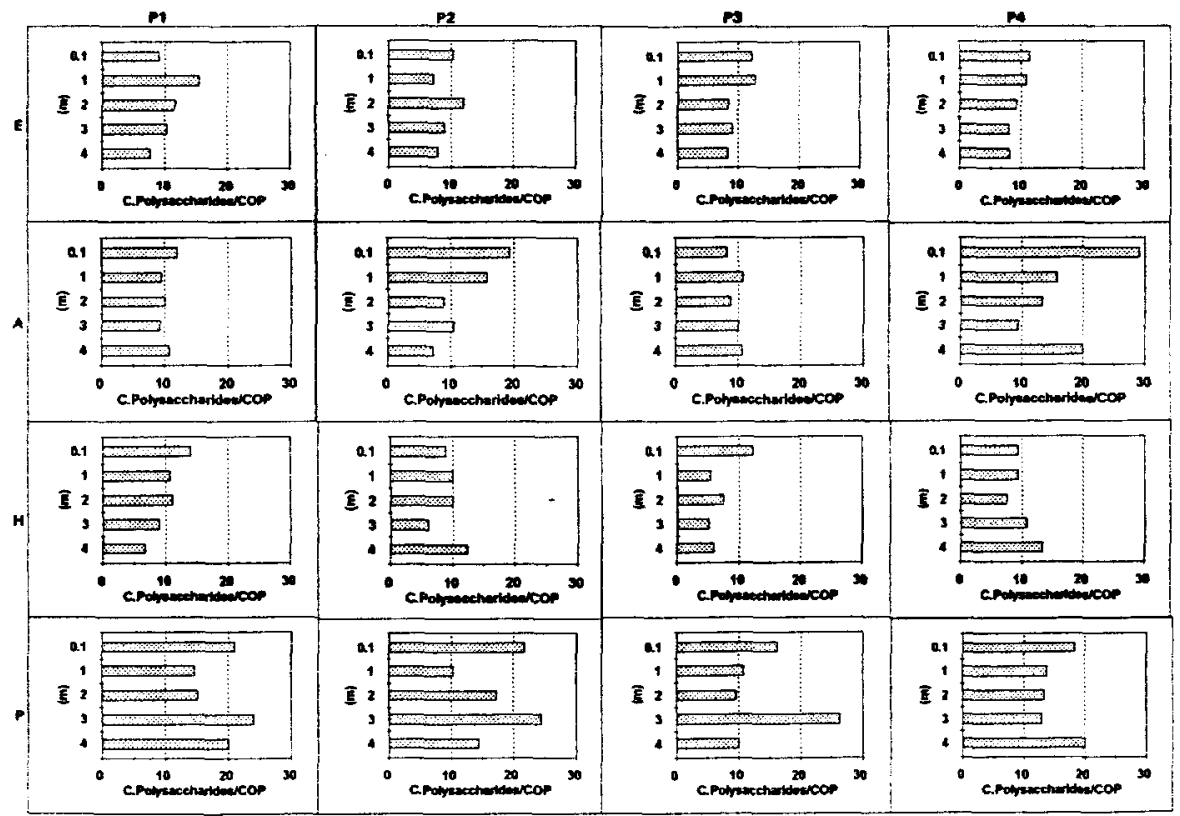

Figure 4 Evolution des teneurs en carbone des polysaccharides rapportées au COP (\%) dans la colonne d'eau au cours des saisons ( $E, A, H$ et $P$ ) sur les quatre stations sélectionnées.

Seasonal changes of the polysaccharide carbon content related to $P O C(\%)$ in the water column at the four selected stations $(E=$ summer, $A=$ autumn, $H=$ winter,$P=$ spring). 


\subsubsection{Les composés phénoliques}

La méthode utilisée permet la détermination des composés phénoliques dérivés de la lignine (composés syringiques, vanilliques et cinnamiques $(S+V+C)$ auxquels appartient une partie relativement stable des composés hydroxybenzyles $(H)$. Une grande abondance de $H$ comparativement au groupe $S+V+C$ autorise à leur attribuer une origine autochtone essentiellement phytoplanctonique ou hydrophytique (HEDGES, et al., 1988 ; SERVE et al., 1997). II est alors possible de distinguer, à partir de ces biomarqueurs, la matière organique aquagène (phytoplanctonique ou algale) et la matière organique pédogène (végétaux supérieurs et humus issus du bassin versant), selon la terminologie de BUFFLE et al. (1987). Dans les suspensions, de faibles quantités de phénols en été (moyenne 2,04 $\mathrm{mg} \cdot \mathrm{H}^{-1}$ ) et particulièrement au printemps (moyenne 1,04 $\mathrm{mg} \cdot \mathrm{H}^{-1}$ ), résultent des conditions de milieu.

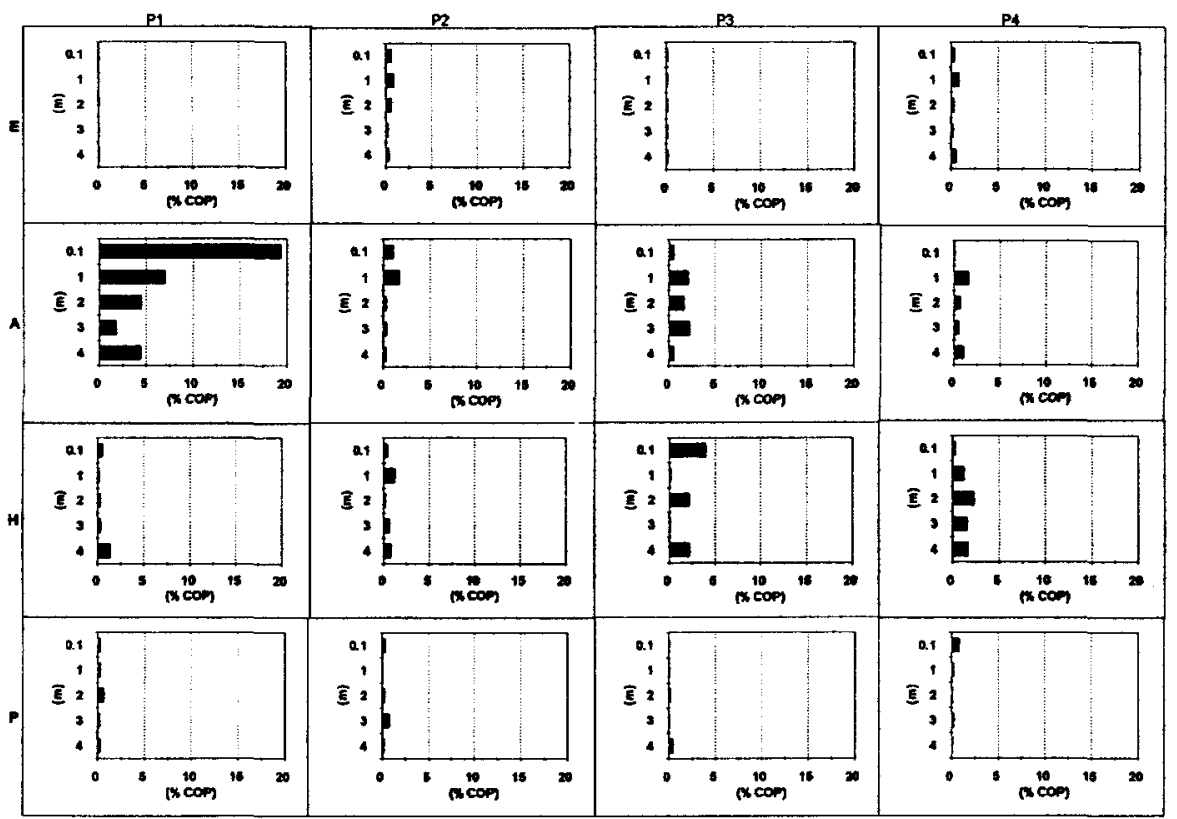

Figure 5 Évolution des teneurs en composés phénoliques rapportées au COP (\%) dans la colonne d'eau au cours des saisons ( $E, A, H$ et $P$ ) sur les quatre stations sélectionnées (analyse CLHP).

Seasonal changes of the phenolic compound content related to POC $(\%)$ in the water column during at the four selected stations (HPLC analysis) ( $E=$ summer, $A=$ autumn, $H=$ winter, $P=$ spring).

En été, les teneurs en phénols rapportées au COP sont assez négligeables (figure 5). En automne et en hiver, les composés phénoliques riches en composés syringiques, vanilliques et cinnamiques $(S+V+C)$ d'origine plutôt pédogène, constituent une part appréciable des apports terrestres introduits dans la lagune par les cours d'eau (tableau 5). Toutefois les composés syringiques, plus fragiles, ne sont pas présents en toute saison. En été et au printemps, les phé- 
nols ont une origine plutôt autochtone. En effet les composés hydroxybenzyles assez abondants au cours de ces saisons (de 0.2 à $0.6 \%$ COP), bien qu'ubiquistes, sont en effet plutôt d'origine autochtone.

\subsubsection{Les classes majeures de composés organiques analysées par pyrolyse}

Les résultats de la pyrolyse exprimés en pourcentages relatifs permettent de préciser dans le matériel en suspension la distribution de classes majeures de composés organiques indicatrices des diverses sources et des effets de dégradation (PUIGBO et al., 1989) (figure 6).

Les hydrocarbures aromatiques, fragments de la pyrolyse et indicateurs de dégradation (BRACEWELL et ROBERTSON, 1981 ; SERES-ASPAX et al., 1985), sont moins abondants en hiver, certainement en rapport avec la fraîcheur de la matière organique mieux conservée dans ces conditions froides, mais ils s'accroissent au printemps.

Le rapport benzène/toluène, qui traduit le degré de condensation des noyaux aromatiques (TRASAR et al., 1994), présente à la station P2 des valeurs en moyenne plus faibles au printemps $(0,18)$ contrairement à l'hiver $(0,75)$, comme sur la station P4, dénotant une plus grande fraîcheur du matériel organique. Sur la station hors table (P4), les valeurs sont généralement plus faibles traduisant une matière organique moins évoluée.

Les composés azotés sont moins abondants au printemps et s'accroissent modérément avec la profondeur en automne et en hiver (azote combiné) sur P2 (figure 6).

L'acétamide et l'indol qui provient du tryptophane, composé fragile, qui sont plus abondants en hiver, traduisent une bonne conservation de la matière organique en rapport avec les conditions froides.

Le rapport acétonitrile/pyrrol, qui traduit le caractère aliphatique ou hétérocyclique des composés azotés, est beaucoup plus fort au printemps notamment sur la station P2 (moyenne 2,8) en rapport avec la reprise de l'activité biologique qui confère à la matière azotée une plus grande aliphaticité. Les valeurs les plus faibles sont observées en janvier 92 (moyenne 0,55 station P2).

Les teneurs en sucres sont plus élevées en été sur P2 du fait de la forte activité biologique estivale (figure 6). Elles sont plus faibles en automne sur P2 et P4, signe de dégradation. Une baisse des sucres est souvent observée avec la profondeur liée à la forte dégradation dans la colonne d'eau.

Le rapport furfural + acide acétique / pyrrol qui traduit l'état de fraicheur de la matière organique (WILSON et al., 1983) décroît avec la profondeur de 1,8 à 0,7 en été, en automne et en hiver (dégradation progressive de la matière organique). Sous table il présente les valeurs les plus fortes en été (moyenne 1,2) et au printemps (moyenne de 1,1) et les plus faibles en automne (moyenne 0,55).

Les sucres aminés représentés par l'acétamide sont assez abondants en été et surtout en hiver, période où les conditions froides peuvent assurer une bonne conservation. 

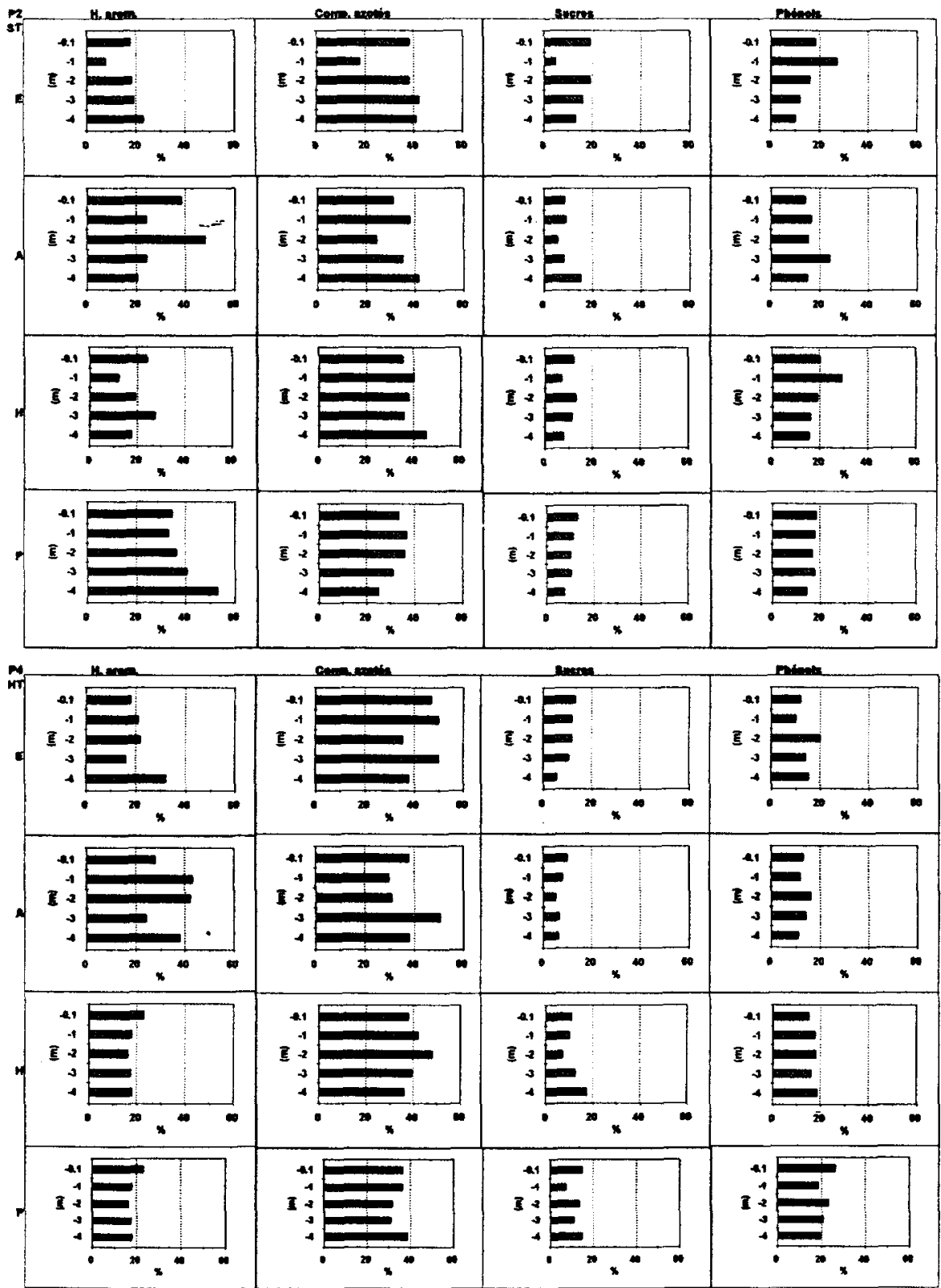

Figure 6 Pourcentages relatifs des classes majeures de composés organiques dans la colonne d'eau au cours des saisons $(E, A, H$ et $P$ ) sur les deux stations sélectionnées sous table P2 et hors table P4 (analyse par pyrolyse).

Seasonal changes in the relative percentages of major organic compound classes in the water column at the two selected stations under table $P 2$ and outside table P4 (PY-GC-MS analysis) ( $E$ = summer, $A=$ autumn, $H=$ winter,$P=$ spring). 
COP $\%$

Sous table (S1)

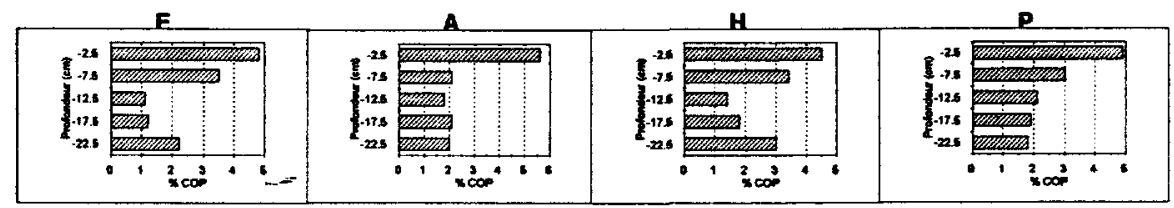

Hors table (\$2)

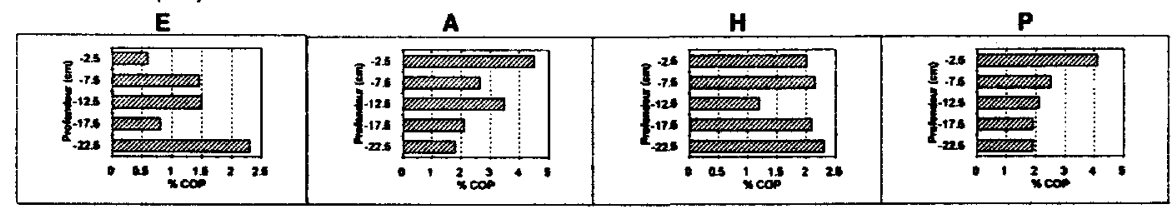

\% C. Poly / COP

Sous table (S1)

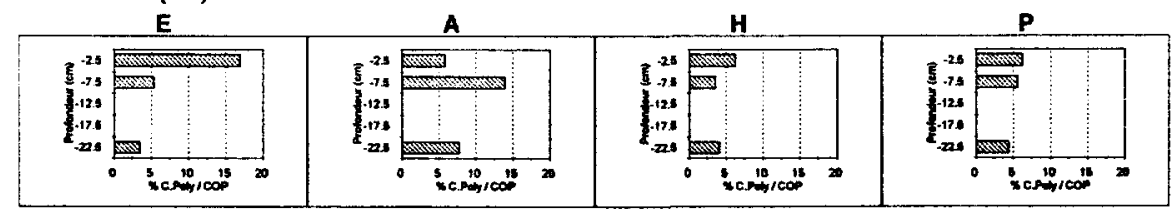

Hors table (\$2)

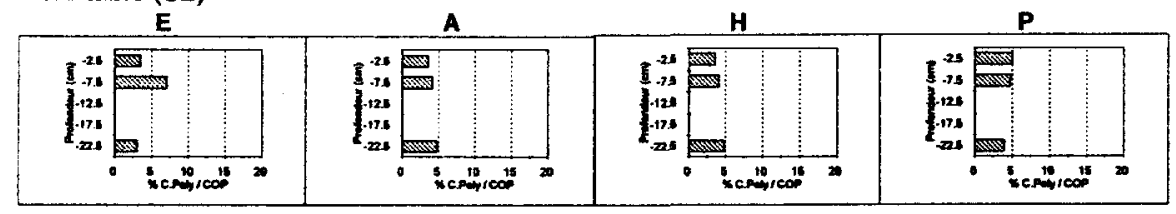

$\%$ Phénols / COP

Sous table (S1)

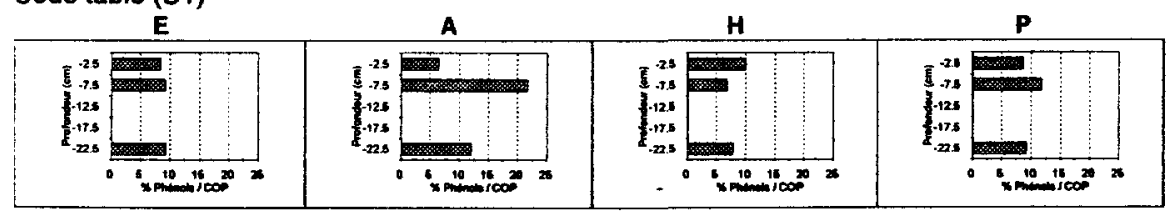

Hors table (S2)
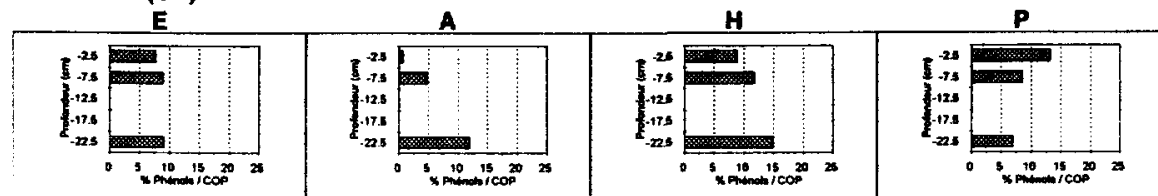

Figure 7 Évolution des teneurs en COP (\%), en polysaccharides et en phénols rapportées au COP (\%) dans les dépôts sélectionnés (sous table S1 et hors table $S 2$ ) au cours des saisons ( $E, A, H$ et $P$ ).

Seasonal changes in POC content (\%), polysaccharide and phenolic contents related to POC (\%) in the selected deposits (under table S1 and outside table $S 4)(E=$ summer, $A=$ autumn, $H=$ winter, $P=$ spring). 


\subsection{Analyse des dépôts}

\subsubsection{Le carbone organique particulaire (COP)}

Sous les tables conchylicoles (station S1) les teneurs en carbone organique sont fortes dans la couche " crème " de surface et décroissent assez rapidement vers la profondeur (figure 7 ). Elles sont moins élevées sur la station hors table (S2), notamment_en été, par suite du caractère plus grossier des dépôts, du milieu plus ouvert, plus oxygéné et de l'éloignement aux tables conchylicoles.

\subsubsection{Les polysaccharides}

Les teneurs en polysaccharides $\left(\mathrm{mg} \cdot \mathrm{g}^{-1}\right)$ suivent sensiblement celles du carbone organique. Elles sont plus élevées et plus variables sous table (de 1,9 à $8,4 \mathrm{mg} \cdot \mathrm{g}^{-1}$ ) et décroissent très rapidement vers la profondeur dans les dépôts. Rapportées au carbone organique elles sont plus élevées en été et en automne sous table (station S1), avec des baisses marquées vers la profondeur en été, moins évidentes en hiver et au printemps (figure 7). La distribution des valeurs est beaucoup plus homogène hors table (station S2) du fait de l'éloignement des tables conchylicoles.

\subsubsection{Les composés phénoliques}

Dans le secteur sous table (station S1), les teneurs en composés phénoliques, plus élevées, décroissent vers la profondeur, pouvant souligner le passage de la forme particulaire à la forme dissoute qui peut être perdue par diffusion. Rapportées au carbone organique (figure 7), elles ne présentent pas d'évolution particulière, mais sont toutefois plus élevées en automne sous table (effet des apports pédogènes liés aux pluies). En terme qualitatif, les phénols d'origine ligneuse $(S+V+C)$ tendent à s'accroître en automne, notamment sur le site hors table (station S2), milieu plus ouvert, mieux exposé aux apports terrigènes entrainés par les pluies. Les phénols hydroxybenzyles d'origine plutôt phytoplanctonique et hydrophytique s'accroissent au contraire en été dans le secteur hors table.

\subsubsection{Les classes majeures de composés organiques analysées par pyrolyse}

Dans l'analyse par pyrolyse, les hydrocarbures aromatiques, indicateurs de dégradation (SERES-ASPAX et al., 1985), relativement abondants dans l'étang, présentent en toutes saisons un accroissement vers la profondeur, indice de la forte dégradation dans les dépôts (diagenèse) (figure 8). Les teneurs semblent plus fortes sur la station hors table (S2), signe d'une plus grande dégradation, peut être due à des conditions plus oxygénées ou à des apports pédogènes plus conséquents en rapport avec des courants plus actifs.

Le rapport benzène / toluène qui rend compte du degré de condensation des noyaux aromatiques (TRASAR-CEPEDA et al. 1994), plus élevé sur la station hors table (moyenne 0,4), s'accroît vers la profondeur dans les dépôts (de 0,3 à 0,5 ), signe d'une dégradation active ou d'une participation moindre de matériel frais.

Les composés azotés, dont l'abondance traduirait la richesse en matériel aquagène, n'offrent pas de tendances marquées, mais le site hors table parait plus riche (azote combiné) (figure 8 ). 
Hydrocarbures aromatiques

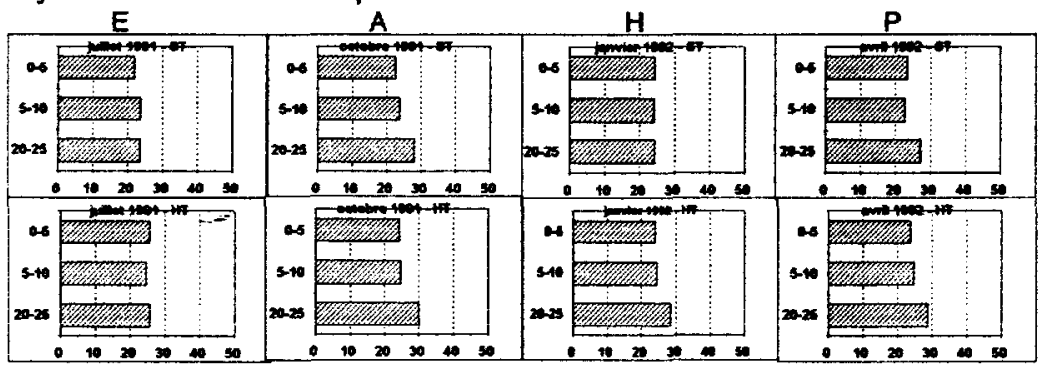

\section{Composés azotés}

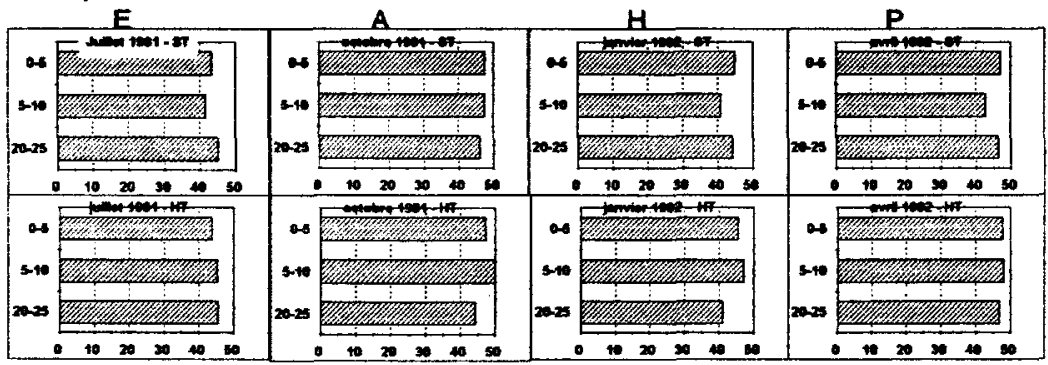

\section{Sucres}

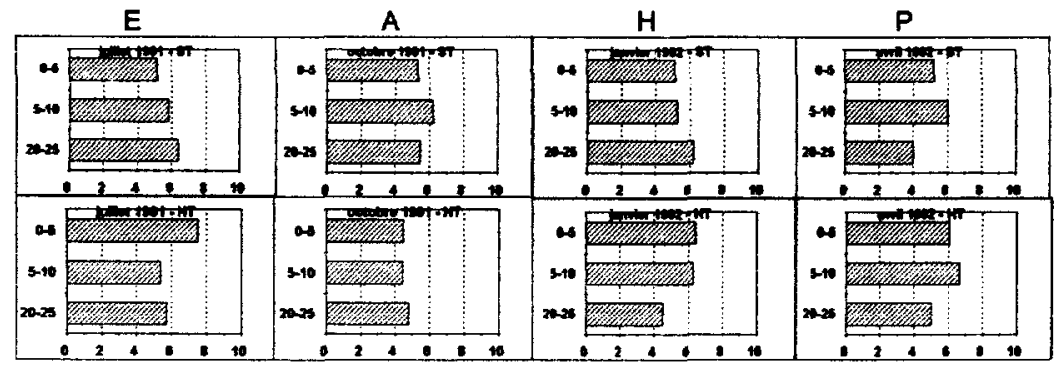

\section{Phénols}

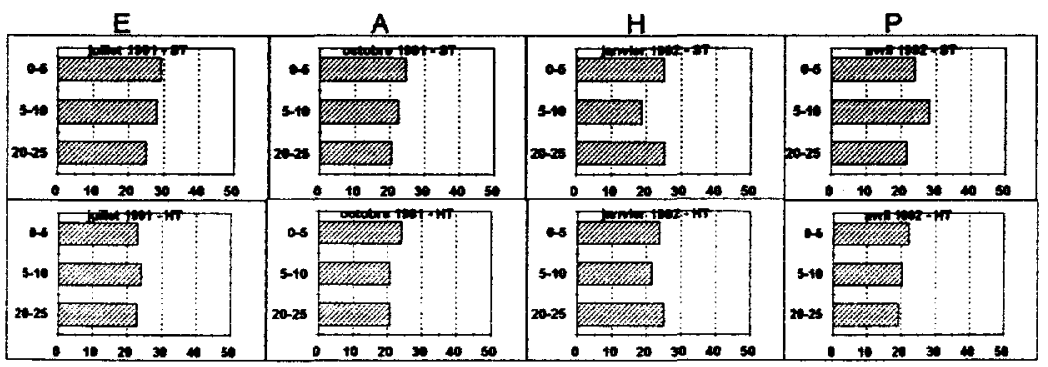

Figure 8 Pourcentages relatifs des classes majeures de composés organiques dans les dépôts sélectionnés (sous table ST et hors table HT) au cours des saisons ( $E, A, H$ et $P$ ).

Seasonal changes in the relative percentages of the major classes of organic compounds in the selected deposits (under table ST and outside table $H T)(E=$ summer, $A=$ autumn, $H=$ winter, $P=$ spring $)$. 
La distribution des sucres paraît assez aléatoire au cours des saisons. Ils tendent plutôt à décroître vers la profondeur sur la station HT S2.

Les phénols, plus abondants sous les tables, décroissent généralement vers la profondeur, certainement du fait du passage de la forme particulaire à la forme dissoute perdue par diffusion.

\subsection{Comparaison des dépôts et des matières en suspension}

La comparaison des caractéristiques du matériel en suspension et des dépôts montre que la moyenne des valeurs du COP est de 20 \% (tableau 1) dans les suspensions et de $3 \%$ dans les dépôts (figure 7 , ce qui rend compte de la forte dégradation de la matière organique dans la colonne d'eau et à l'interface eau-sédiment.

Les polysaccharides sont plus abondants dans les suspensions (moyenne $6 \%$ ) que dans les dépôts $(0,5 \%)$.

Les teneurs en composés phénoliques $\left(\mathrm{mg} \cdot \mathrm{g}^{-1} ; \mathrm{CLHP}\right)$ sont également plus fortes dans les suspensions.

Certains phénols comme les composés vanilliques sont mieux conservés dans les dépôts comparativement à d'autres plus sensibles à la dégradation comme les composés syringiques.

Dans l'analyse par pyrolyse, la matière organique des dépôts est plus riche en hydrocarbures aromatiques que celle des suspensions, traduisant par là une plus forte dégradation du matériel organique.

Les composés azotés sont plus abondants dans les dépôts avec des valeurs plus constantes (azote combiné). Le rapport acétonitrile / pyrrol qui traduit la relation entre l'azote aliphatique et l'azote hétérocyclique paraît légèrement plus élevé en été et en automne dans les dépôts sous table où il décroît vers la profondeur.

Les sucres sont bien plus abondants dans les suspensions que dans les dépôts en rapport avec la plus grande fraîcheur du matériel organique.

Le rapport furfural+acide acétique/pyrrol, indice de l'état de fraîcheur de la matière organique, est également beaucoup plus élevé dans les suspensions, notamment en été (moyenne 1,2) que dans les dépôts (moyenne 0,16 ).

Les phénols sont plus abondants dans les dépôts que dans les suspensions, soulignant la meilleure conservation de ces composés résistants à la dégradation.

Enfin les sucres aminés, comme les sucres, présentent des teneurs élevées dans les suspensions, indice de la fraîcheur du matériel organique.

La quantité de matière organique incorporée en fin de course dans les dépôts superficiels dépend de l'équilibre entre un matériel très labile et frais présent dans la colonne d'eau et une fraction humique condensée accumulée dans les dépôts associée aux éléments figurés organiques. Les sédiments intègrent tous les apports qui se produisent dans la colonne d'eau et introduisent alors un effet de mélange en rapport avec la dégradation active (SIGLEO et al., 1982).

La matière organique constitue à la fois la source et le puits des sels nutritifs. Sous les tables conchylicoles, en période estivale, les quantités de phos- 
phore et d'azote s'accroissent dans les dépôts (MESNAGE, 1994) en rapport avec l'accumulation de la matière organique (fèces d'huîtres) et la dégradation active. Ces effets sont surtout sensibles dans les couches superficielles.

Par ailleurs, les variations des conditions de milieu au cours de l'année se traduisent dans l'accroissement des concentrations en $P$ lié au fer en été en rapport avec des conditions plus oxygénées liées à la photosynthèse et, au ' contraire, une diminution du $\mathbf{P}$ lié au calcium du fait de la baisse du pH (abondance du CO2 et de la matière dissoute) (MESNAGE, 1994). Chaque forme du phosphore est donc sensible aux facteurs environnementaux qui dépendent souvent du taux et de la nature de la matière organique. Les enrichissements en nutriments sont plus marqués sous la table conchylicole du fait de la sédimentation des fèces et des processus actifs de dégradation. Les teneurs sont beaucoup plus constantes sous la station éloignée des tables conchylicoles (S2). La matière organique conditionne donc la distribution des sels nutritifs, à divers degrés, ceci en fonction de la situation par rapport aux tables conchylicoles qui, elle-même, contrôle son abondance dans la colonne d'eau et les dépôts.

\section{4 - CONCLUSION}

Cette étude a permis de préciser, dans une lagune méditerranénne, l'origine et le comportement de la matière organique au cours des saisons en fonction de la variabilité climatique et de l'activité biologique liée pour partie à l'activité conchylicole.

Le fonctionnement de cet écosystème varie avec le temps au niveau de l'importance et de la nature de la matière organique. Les conclusions essentielles de l'étude sont portées sur la figure 9 qui montre, suivant les saisons, l'évolution des caractères majeurs de la matière organique dans la colonne d'eau et les dépôts de l'écosystème considéré.

- En été, une forte production biologique accroît la quantité de matériel en suspension. La teneur élevée en matière organique dissoute est en rapport avec les processus d'excrétion et la dégradation de la matière organique particulaire. Les teneurs en sucres sont assez élevées et basses en phénols qui sont plutôt aquagènes. Des processus de décantation entraînent une stratification de la colonne d'eau avec accumulation de la matière organique en profondeur (conditions de calme : absence de courants).

- En automne, la productivité biologique est plus faible avec une matière en suspension moins abondante. Les processus de dégradation sont très actifs (dégradation des sucres) comme le recyclage du carbone organique dissous. Les teneurs en composés phénoliques d'origine pédogène s'accroissent en rapport avec les pluies.

- En hiver, l'activité biologique est très réduite avec pour résultat une matière en suspension très faible. Les teneurs en matière organique et en sucres sont faibles. La présence de composés fragiles comme l'indol rend compte d'une bonne conservation de la matière organique liée aux conditions froides, matériel organique qui n'a pas été dégradé en automne. L'homogénéi- 


\begin{tabular}{|c|c|c|c|c|}
\hline \multicolumn{5}{|c|}{ Fonctlonnoment schomatlque de l'ócosystome lagunalre au cours d'une annde } \\
\hline Eté & Automne & Hiver & Printemps & Caractónstique principale \\
\hline $\begin{array}{l}\text { - Forte production } \\
\text { biologique }\end{array}$ & $\begin{array}{l}\text { - Réduction den } \\
\text { l'activité biologique }\end{array}$ & $\begin{array}{l}\text { - Activité biologique } \\
\text { trés réduite }\end{array}$ & $\begin{array}{l}\text { Reprise de l'activité } \\
\text { biologique }\end{array}$ & 8 \\
\hline $\begin{array}{l}\text { Stratification de la } \\
\text { colonne d'eau } \\
\text { Accumulation de la } \\
\text { matiere organique } \\
\text { en profondeur. }\end{array}$ & $\begin{array}{l}\text { - Légère } \\
\text { stratification de la } \\
\text { colonne d'eau }\end{array}$ & $\begin{array}{l}\text { Homogénéisation de } \\
\text { la colonne d'eau }\end{array}$ & $\begin{array}{l}\text { Homogénéisation de la } \\
\text { colonne d'eau }\end{array}$ & \\
\hline $\begin{array}{l}\text { - Abondance de } \\
\text { matiere organique } \\
\text { dissoute (COD) } \\
\text { - Dégradation des } \\
\text { sucres avec la } \\
\text { profondeur }\end{array}$ & $\begin{array}{l}\text { Degradation active } \\
\text { et recyclage du } \\
\operatorname{COO}\end{array}$ & $\begin{array}{l}\text { Charge organique } \\
\text { réduite }\end{array}$ & - Abondance des sucres & \\
\hline & $\begin{array}{l}\text { - Apports terrigénes } \\
\text { - Abondance des } \\
\text { phénols ligneux }\end{array}$ & $\begin{array}{l}\text { - Apports terrigenes } \\
\text { - Abondance des } \\
\text { phenols ligneux }\end{array}$ & & \\
\hline Sédiment & 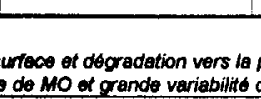 & ( & the & - \\
\hline
\end{tabular}

Figure 9 Diagramme synthétique du fonctionnement de l'écosystème lagunaire au cours des saisons (colonne d'eau et dépôts). Summary chart showing the functioning of the lagoon during the different seasons (water column and deposits). 
sation de la colonne d'eau résulte de l'action des courants. Les apports pédogènes sont également conséquents.

- Au printemps, la charge en suspension est assez réduite. Mais la reprise de l'activité biologique est responsable de la fraîcheur du matériel et de la plus grande abondance des sucres.

Les résultats montrent bien l'impact de l'ostréiculture dans l'accroissement ' de le matière orgañíque dans la colonne d'eau et les sédiments sous les tables conchylicoles. La sédimentation organique et les processus dégradatifs sont donc plus ou moins actifs en fonction de la position des stations et de leurs caractéristiques. Des différences s'observent en effet suivant les stations. Si sur les profils la distribution des divers paramètres est assez voisine sous les stations sous table, ils différent dans les couloirs. La production biologique est alors moindre et le milieu plus oxygéné. II en résulte une plus grande constance dans la distribution des paramètres biogéochimiques. Certaines de ces particularités sont également retrouvées au niveau des dépôts.

Comme le signale BIOTEAU (1993) les effets quantitatifs des huittres et des dépôts dans le cycle du carbone semblent moins efficaces que les processus de minéralisation dans la colonne d'eau en cours d'année. Ces derniers favorisent la transformation de la matière organique particulaire en matière dissoute. De plus, les apports extérieurs, pédogènes en automne et en hiver, jouent certainement un rôle actif dans le fonctionnement de l'écosystème.

La comparaison de ces résultats avec les données obtenues sur les nutriments dans la colonne d'eau et les dépôts (MESNAGE, 1994) permet de corréler l'abondance des sels nutritifs avec les processus de formation et de dégradation de la matière organique et de reconnaître leurs effets réels dans les phénomènes d'eutrophisation. La période estivale d'accumulation de matière organique favorable à ces processus coïncide d'ailleurs avec des quantités accrues de phosphore et d'azote. Chaque forme du phosphore est sensible aux facteurs environnementaux qui souvent dépendent du taux et de la nature de la matière organique. Celle-ci peut donc conditionner le comportement des sels nutritifs comme le relargage hivernal du phosphore ou au contraire sa rétention estivale.

\section{RÉFÉRENCES BIBLIOGRAPHIQUES}

BIOTEAU H., 1993 Modélisation de l'écosystème de l'étang de Thau : rôles du sédiment et des huîtres cultivées sur le cycle de l'azote. Rapp. DEA, Université de Paris 6, Paris, $47 \mathrm{p}$.

BRACEWELL J.M. et ROBERTSON G.W., 1981. Analytical potential of pyrolysis products derived from soil organic matter. Analyt. Proc., 18, 532-535.

BUFFLE J., ZALI O., ZUMSTEIN J. et De VITRE R., 1987. Analytical methods for the direct determination of organic and inorganic species: seasonal changes of iron, sulfur and pedogenic and aquagenic organic constituents in the eutrophic lake Bret, Switzerland. Sci. Total Environ., 64, 41-59.

CHAPELLE A., 1993. Modèles des cycles de l'azote et du phosphore à l'interface eausédiment-Application à l'étang de Thau. Rapp. interne IFREMER DEL Brest93.17-56 p. 
CHARRIERE B., SANCHO A., SERVE L., COMBAUT G., GADEL F. et PIOVETTI L., 1986. Les composés phénoliques dans un écosystème lagunaire méditerranéen : végétaux aquatiques, eaux, sédiments. Journées Internationales d'Etudes du Groupe Polyphénols, Montpellier.

DESLOUS-PAOLI -J:M., 1991. SORNIN J.M., DURAND F., CHEVALIER M., LAGABRIELLE R., PELISSIER M., TOURNIER H. et JUGE C. Cartographie et caractéristiques des sédiments superficiels du secteur conchylicole de l'étang de Thau. Rapp. IFREMER, DRO/EM 91.03, 61 p.

FRISONI et CEJPA A.M., 1985. La malaïgue dans les étangs littoraux du LanguedocRoussillon. Rapp. CEPRALMAR ; $48 \mathrm{p}$.

GADEL F., 1994. Évolution saisonnière de la matière organique dans les suspensions et les sédiments de l'étang de Thau : distribution et nature. Journées OXYTHAU, Sète.

GADEL F. et SERVE L, 1994. Evolution saisonnière de la matière organique dans un écosystème saumâtre : l'étang de Thau. Congrès "De l'étude à l'aménagement des eaux lagunaires et côtières ». European Training Sessions, Sète.

GALLAL| T., 1972. Étude statique et dynamique des polysaccharides et des aminopolysaccharides au cours de l'humification. Th.Doct. Univ. Nancy, 93 p.

HARTLEY R.D. et BUCHAN, 1979. High-performance liquid chromatography of phenolic acids and aldéhydes derived from plants or from the decomposition of organic matter in soil. J. chromatogr., $180,139-143$.

HEDGES J.I. \& PARKER P.L., (1976). Landderived organic matter in surface sediments from the Gulf of Mexico. Geochim. Cosmochim. Acta, 40, 1019-1029.

HEDGES J.I., \& MANN D.C., (1979). The characterization of plant tissues by their lignin oxidation products. Geochim. Cosmochim. Acta, 43, 1809-1818.

HEDGES J.I. et ERTEL J.R., 1982. Characterization of lignin by gas capillary chromatography of cupric oxide oxidation products. Anal. Chem., 54, 174-178.

HEDGES J.I., CLARK W.A. et COWIE G.L., 1988. Organic sources to the water column and surficial sediments of a marine bay. Limnol. Oceanogr., 33, 11161136.

JOUFRE D. et AMANIEU M., 1991. Synthèse des résultats du programme ECOTHAU. Université Sci.Tech. Languedoc Montpellier II.

MESNAGE V., 1994. Contribution à l'étude de la mobilité des formes de phosphate à l'interface eau-sédiment dans des écosystèmes lagunaires. Th. Doct. Univ. Montpellier I, 253.

MILLET B., 1989. Fonctionnement hydrodynamique du bassin de Thau. Validation d'un modèle numérique de circulation (Programme ECOTHAU). Oceanol. Acta, 12, 37-46.

PUIGBO A., GADEL F., ALCANIZ J.M. et COMELLAS L., 1989. PY-GC-MS analysis of organic matter in suspended material and deposits of the submarine delta of the Rhône river (France). Sci. Total Environ. 81-82, 71-80.

SENEGAS M. et DOMANGE M., 1990. Les milieux lagunaires. Importance, originalité, diversité, richesse, évolution. L'État au Présent $n^{\circ} 16,6-7$.

SERES-ASPAX A., ALCANIZ-BALDELLOU J.M. et GASSIOT M., 1985. Application of pyrolysis-gas chromatography to the study of the composting process of barley straw and pear-tree wood. J. Analyt. Appl. Pyrolysis, 8, 415-426.

SERVE L., PIOVEITI L. et LONGUEMARD N., 1983. Dosage des acides et des aldéhydes phénoliques par chromatographie liquide haute performance : analyse com- parative des sols de haute montagne et de leur végétation. J. Chromatogr., 259, 319-338.

SERVE L., GADEL F., CHARRIERE B.et BLAZI J.L., 1993. Biogeochemical characters of organic matter in the water column of a brakish ecosystem : the Thau lagoon. Relations with sediments. $X I$ International Symposium on Environmental Biogeochemistry, Salamanque.

SERVE L., ICOLE M. et BLAZI.J.L., 1997. Les monomères phénoliques dérivés des lignines dans les sédiments d'une carotte longue du lac du BOUCHET (Massif Central, France) et la reconstitution des paléoenvironnements. Bull. Soc. Géol. Fr, 168 (4), 507-520. 
SIGLEO A.C., HOERING T.C. et HELZ G.R., 1982. Composition of estuarine colloidal material : organic components. Geochim. Cosmochim. Acta, 46, 1619-1626.

TRASAR-CEPEDA M.C., CECCANTI B., LEIROS M.C., CALCINAI M. et GIL-SOTRES F., 1994. Characterization of organic matter in lignite minesoils at various ages by pyrolysis-gas chromatography. In Humic Substances in the Global Environment and Implications on Human Health. N.Senesi and T.M. Miano eds : 707-712.
WALSH G.E., 1966. Studies on dissolved carbohydrates in Cape Cod waters. III Seasonal variations in Oyster Pond and Wequaquet Lake, Massachusetts. Limnol. Oceanogr., 11, 249-256.

WILSON M.A., PHILP R.P., GILLAM A.N., GILBERT T.D. et TATE K.R., 1983. Comparison of the structures of humic substances from aquatic and terrestrial sources by pyrolysis-gas chromatography-mass spectrometry. Geochim. Comochim. Acta, 47, 497-502. 